\title{
A high-temperature superconducting monolithic microwave integrated Josephson down-converter with high conversion efficiency
}

J. Du, T. Zhang, Y. J. Guo, and X. W. Sun

Citation: Appl. Phys. Lett. 102, 212602 (2013); doi: 10.1063/1.4808106

View online: http://dx.doi.org/10.1063/1.4808106

View Table of Contents: http://aip.scitation.org/toc/apl/102/21

Published by the American Institute of Physics

\section{Articles you may be interested in}

A self-pumped high-temperature superconducting Josephson mixer: Modelling and measurement Journal of Applied Physics 111, 053910 (2012); 10.1063/1.3691191

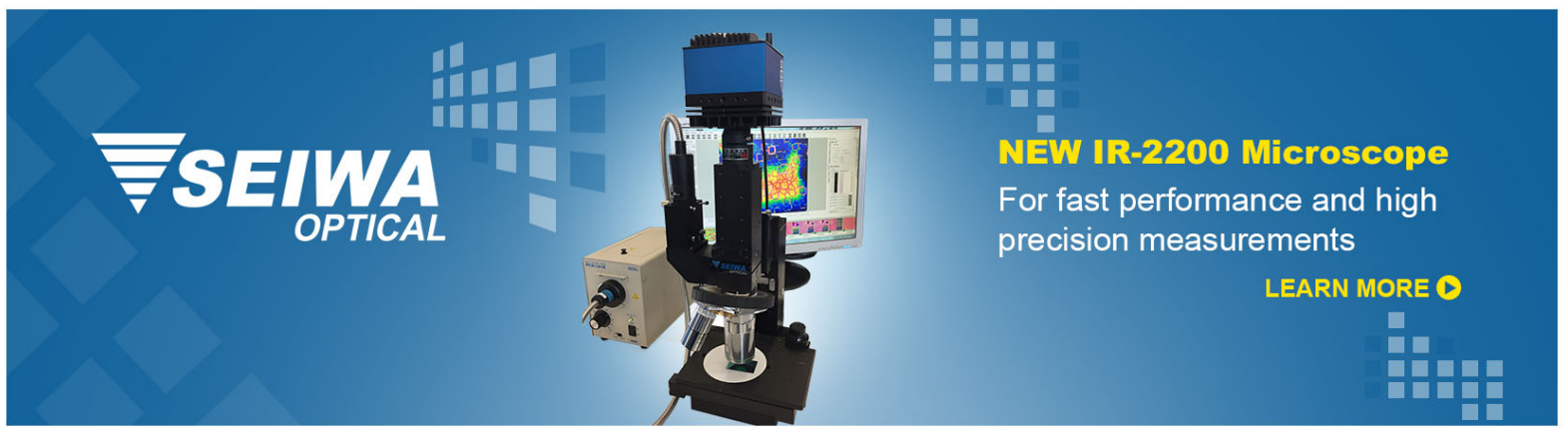




\title{
A high-temperature superconducting monolithic microwave integrated Josephson down-converter with high conversion efficiency
}

\author{
J. Du, ${ }^{1}$ T. Zhang, ${ }^{2,3}$ Y. J. Guo, ${ }^{2}$ and X. W. Sun ${ }^{3}$ \\ ${ }^{1}$ CSIRO Materials Science and Engineering, PO Box 218, Lindfield, NSW 2070, Australia \\ ${ }^{2}$ CSIRO ICT Centre, PO Box 76, Epping, NSW 1710, Australia \\ ${ }^{3}$ Shanghai Institute of Microsystem and Information Technology, CAS, China
}

(Received 4 March 2013; accepted 10 May 2013; published online 30 May 2013)

\begin{abstract}
A compact high- $\mathrm{T}_{\mathrm{c}}$ superconducting monolithic microwave integrated circuit Josephson downconverter is presented. The circuit consists of a single Josephson junction mixer, a bandpass filter, a lowpass filter, and a resonator for local oscillator fabricated on a single $10 \mathrm{~mm} \times 20 \mathrm{~mm}$ chip of $\mathrm{YBa}_{2} \mathrm{Cu}_{3} \mathrm{O}_{7-\mathrm{x}}$ film on $\mathrm{MgO}$ substrate. The down-converter demonstrates superior performance in terms of conversion efficiency, dynamic range, linearity, and low local oscillator power with stable operation from 20 to $77 \mathrm{~K}$. A maximum conversion gain of $-4.7 \mathrm{~dB}$ was measured at $20 \mathrm{~K}$ and $-12.8 \mathrm{~dB}$ at $70 \mathrm{~K}$. [http://dx.doi.org/10.1063/1.4808106]
\end{abstract}

High-temperature superconducting (HTS) thin films offer unique properties, which can be utilised for a variety of high-frequency device applications. One important property is the exceptionally low level of microwave losses at temperatures attainable with low power cryocoolers. Highperformance HTS passive devices like filters and resonators with exceptionally high-Q and low loss have been well developed and applied in wireless base stations. ${ }^{1-3}$ The second important property of the HTS films is related to the AC Josephson effect. The extreme nonlinearity of the currentvoltage (I-V) relationship of Josephson junctions makes them ideal for applications in active microwave devices, such as oscillators, amplifiers, and mixers. However, the Josephson junction based microwave active devices are less mature due to the rather challenging HTS junction technology. It is even a greater challenge to integrate the HTS passive and active components onto a single chip to form a functional HTS circuit. Only few attempts of integrating HTS components on-chip were trialled. ${ }^{4,5}$ A hybrid configuration of HTS passive components with semi-conducting active devices has been typically employed in HTS RF frontend receiver circuits. ${ }^{1,2}$ Such a compromise cannot exploit the full potential of superconductive electronics. Developing monolithic HTS circuits is a critical step for the application of the HTS technology to terrestrial and space communication systems.

In previous work, we reported a monolithic HTS frequency down-converter ${ }^{6}$ comprising a self-pumped Josephson heterodyne Resistive-SQUID (R-SQUID) oscillator-mixer device, ${ }^{7-10}$ a bandpass filter, ${ }^{11}$ and a lowpass filter. ${ }^{12}$ The self-pumped Josephson R-SQUID mixer has an oscillation linewidth (in the order of $\mathrm{MHz}$ ) too broad for applications in telecommunication systems despite a $10^{3}$ order magnitude improvement compared to that of a single Josephson junction oscillator. The linewidth broadening degrades the phase noise. In addition, the maximum achievable conversion gain $(-15 \mathrm{~dB})$ was limited by the small available power from the internal oscillator (up to $-70 \mathrm{dBm}$ or $100 \mathrm{pW}$ ). Higher conversion efficiency in the mixer is desired for the receiver systems.

In this work, we consider a different approach; a single Josephson junction mixer externally pumped and integrated with other HTS passive components to form a HTS monolithic microwave integrated circuit (MMIC) down-converter for RF receiver front-ends. The aim is to improve the conversion efficiency and linewidth (for low phase noise) while retaining the features of coupling efficiency, compactness, low loss, and low power consumption that an MMIC could offer. For the performance comparison, we used the same designs of the filters ${ }^{11,12}$ for the RF input and the intermediate frequency (IF) output but added an additional HTS resonator for the local oscillator (LO) port to stop the RF leakage into the LO port. The full evaluation of the described monolithic HTS down-converter is given in this paper.

A Josephson junction is a highly nonlinear device that converts a weak RF signal to an IF signal under the action of a local oscillator such as $f_{\mathrm{IF}}=f_{\mathrm{RF}}-f_{\mathrm{LO}}$ (single-sideband mixer). The operation of a Josephson mixer can be analysed using a resistive shunt Josephson (RSJ) model (see, for example, Refs. 13 and 14). An important measure of a mixer or frequency down-converter is the conversion efficiency, which is the ratio of the power produced at the $\mathrm{IF}, P_{\mathrm{IF}}$, to the input power at the RF, $P_{\mathrm{RF}} ; \eta=P_{\mathrm{IF}} / P_{\mathrm{RF}}$. Other factors such as frequency response, dynamic range, linearity, DC bias and LO bias points, and LO power consumption are also important for application of the device.

For a resistively shunt Josephson junction, the conversion efficiency can be conveniently expressed in terms of experimental measurable quantities ${ }^{13}$

$$
\eta=C_{\mathrm{IF}}\left(R_{\mathrm{D}} / R\right) \alpha^{2},
$$

where $\mathrm{C}_{\mathrm{IF}}$ is the output coupling efficiency $(\leq 1), R_{\mathrm{D}}$ is the dynamic resistance (the differential slope of the I-V graph) at the bias point. $R$ is the shunt resistance in LTS (lowtemperature superconducting) Josephson junctions and the normal resistance, $R_{\mathrm{n}}$, in the case of intrinsically resistiveshunted HTS junctions. $\alpha$ is a dimensionless parameter defined by

$$
\alpha=\frac{\partial\left(I_{\mathrm{c}} / I_{\mathrm{c} 0}\right)}{\partial\left[\left(8 P_{\mathrm{LO}} / R\right)^{1 / 2}\right]},
$$


where $P_{\mathrm{LO}}$ is the available power from the LO source. $I_{\mathrm{c}}$ is the junction critical current at operating condition (suppressed by LO) and $I_{\mathrm{c} 0}$ is the value at $P_{\mathrm{LO}}=0$. A positive conversion gain $(>0)$ has been observed in LTS Josephson mixers. ${ }^{15}$ For most HTS Josephson mixers, however, the reported conversion gain is below $-10 \mathrm{~dB}$ and a maximum conversion gain of $-8 \pm 4 \mathrm{~dB}$ was observed by Butler et al. at $25 \mathrm{~K}$ and at comparable frequency. ${ }^{16}$

Fig. 1(a) shows the layout of the described monolithic HTS down-converter consisting of a single $\mathrm{YBa}_{2} \mathrm{Cu}_{3} \mathrm{O}_{7-\mathrm{x}}$ (YBCO) Josephson mixer, 6.7-8.7 GHz band-pass filter for the RF input, a low-pass filter with cut-off frequency at $4 \mathrm{GHz}$ for the IF output, a $5 \mathrm{GHz}$ resonator for the LO port. The addition of the resonator is to eliminate RF power leakage. Fig. 1(b) is a photograph of the packaged HTS circuit chip with a printed circuit board (PCB) containing DC biasing lines and a RC isolation circuit. The $1 \mathrm{k} \Omega$ resistors and the RF block network are used on the biasing lines for eliminating RF signals. Bonding wires are used for the connections between the HTS device
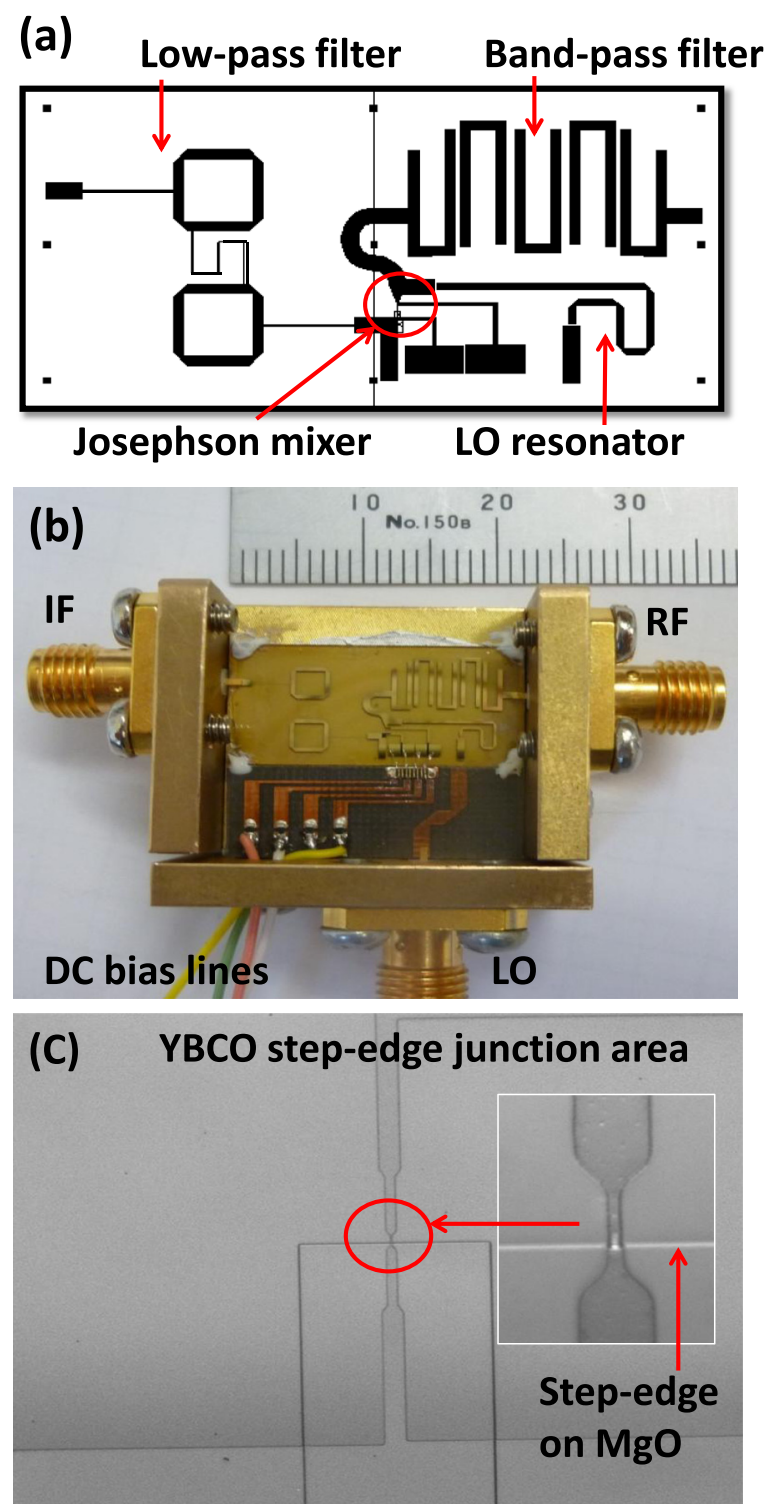

FIG. 1. (a) The layout of the monolithic HTS Josephson down-converter, (b) a photograph of the packaged HTS circuit, and (c) a close-up view of the Josephson junction where a $2 \mu \mathrm{m}$ wide YBCO micro strip-line across a step-edge created on the $\mathrm{MgO}$ substrate. and the PCB board. The total size of the packaged module is approximately $2.6 \mathrm{~cm} \times 2.6 \mathrm{~cm} \times 1.6 \mathrm{~cm}$, which is very compact. Fig. 1(c) is the close-up view of the YBCO step-edge grain boundary junction (GBJ).

The HTS chip was fabricated on $20 \mathrm{~mm} \times 10 \mathrm{~mm}$ $\times 0.5 \mathrm{~mm} \mathrm{MgO}$ substrate using the CSIRO- developed YBCO step-edge junction technology. ${ }^{17,18}$ Briefly, the steppattern was created on the $\mathrm{MgO}$ substrate using photolithographic and $\mathrm{Ar}$ ion beam etching techniques. A $220 \mathrm{~nm}$ YBCO film with a $50 \mathrm{~nm}$ in-situ Au film was deposited on the $\mathrm{MgO}$ substrate by Theva $\mathrm{GmbH}$. The $\mathrm{YBCO} / \mathrm{Au}$ film was then patterned and etched using the photolithographic and $\mathrm{Ar}$ ion beam etching techniques. The in-situ Au film was removed from the junction area. A layer of Au film was deposited on the back of the chip. One of the major advantages of step-edge junctions is the flexibility of positioning the junction anywhere on the substrate, simplifying device design for the on-chip HTS circuit. The junction parameters can be tuned to some degree to improve the device performance. The fabricated chip was packaged in a copper thermal housing and cooled down on a pulse-tube cryocooler. No additional RF and magnetic shielding were used for the sample on the cryocooler. A battery-operated current source was used to bias the Josephson mixer. The microwave RF signal generated by an Agilent Technologies E8257D signal generator was applied to the RF port of the MMIC chip. A low power $5 \mathrm{GHz}$ RF signal was applied to the LO port. The down-converted IF outputs were measured using an Agilent E4407B spectrum analyser. No amplifier was used for the output signal.

Fig. 2 shows the DC current-voltage (I-V) characteristics of the Josephson junction measured at different temperatures, without applied RF signal. It exhibits a typical RSJ behaviour without a hysteresis. The junction normal resistance, $R_{n}$, determined from the linear slope, is around $20 \Omega$, which is very high. The $I_{\mathrm{c}}$ is $106 \mu \mathrm{A}$ at $40 \mathrm{~K}$ and $9 \mu \mathrm{A}$ at $77 \mathrm{~K}$. The exceptionally high $R_{\mathrm{n}}$ value is ideal for the Josephson mixer as it improves the impedance match between the junction and the $50 \Omega \mathrm{RF}$ circuit thus increases the coupling efficiency of the RF power into the junction $\left(P_{\mathrm{RFJ}}=I_{\mathrm{RF}}^{2}\left(R_{\mathrm{n}} / 2\right)\right.$, where $I_{\mathrm{RF}}$ is the applied RF current). The dynamic resistance, $R_{D}=d V / d I$, above the $I_{\mathrm{c}}$, increases with decreasing the temperature.

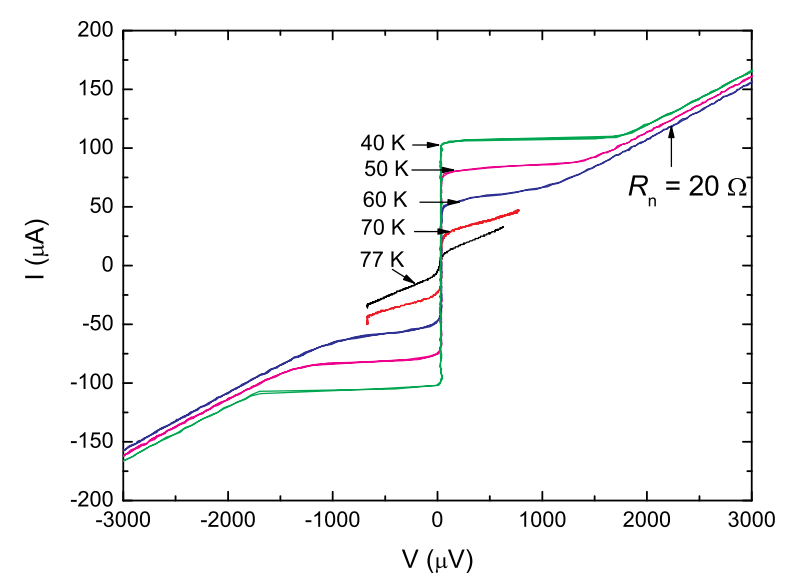

FIG. 2. The I-V characteristics of the step-edge junction at different temperatures. 

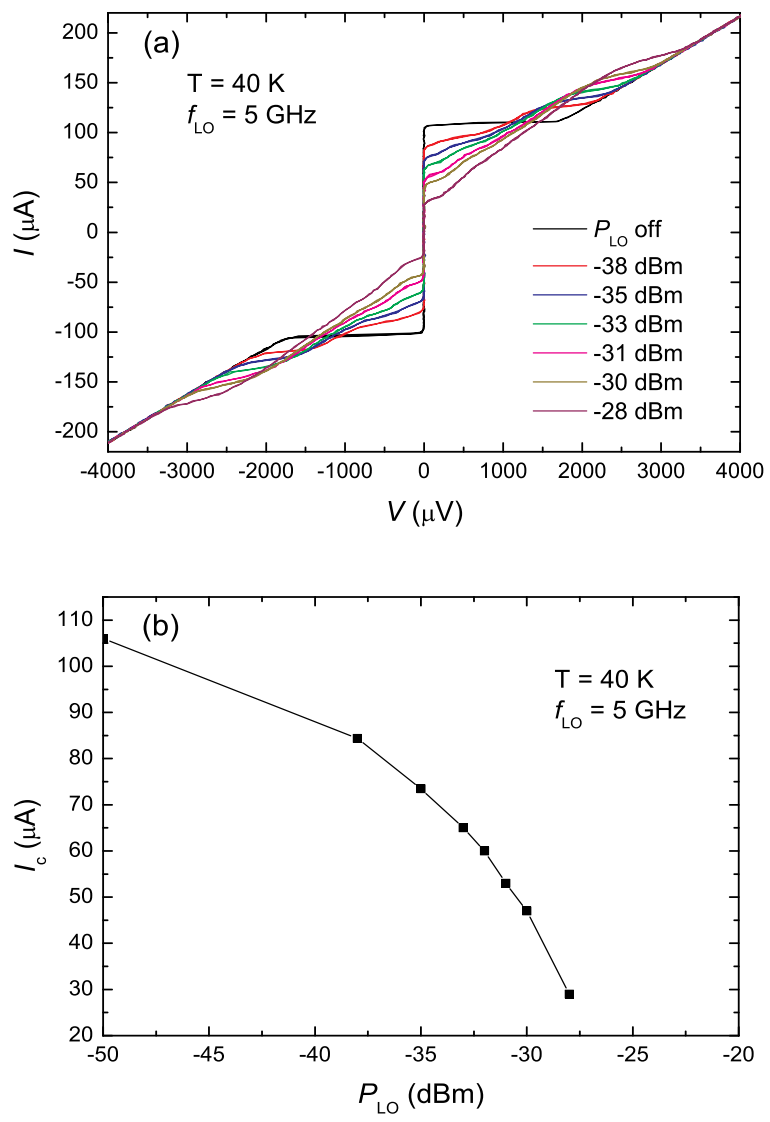

FIG. 3. (a) I-V curves at different LO power levels; and (b) the $I_{\mathrm{c}}$ value versus $P_{\mathrm{LO}}$ at $40 \mathrm{~K}$.

Differentiation of the $\mathrm{I}-\mathrm{V}$ at $40 \mathrm{~K}$ yields a maximum $R_{D} \approx 60$ $\Omega\left(P_{\mathrm{LO}}=0\right)$, which is quite high for an HTS junction. A high $R_{\mathrm{D}}$ value is desired for increasing the IF output.

Fig. 3(a) shows the I-V curves at $40 \mathrm{~K}$ for different levels of the LO power. Unsuppressed junction critical current $I_{\mathrm{c} 0}=106 \mu \mathrm{A}$, and the $I_{\mathrm{c}}$ is suppressed by the input LO signal. No Shapiro step is resolved from the graphs due to very low frequency and, therefore, small voltage steps $\left(f / V=0.4836 \mathrm{GHz} / \mathrm{V}\right.$, giving $\Delta \mathrm{V} \approx 10 \mu \mathrm{V}$ for $\left.f_{\mathrm{LO}}=5 \mathrm{GHz}\right)$. Fig. 3(b) plots out the $I_{\mathrm{c}}$ value against the LO power. The best conversion gain was obtained at $P_{\mathrm{LO}} \sim-32 \mathrm{dBm}$ (at $40 \mathrm{~K}$ ), where $I_{\mathrm{c}} / I_{\mathrm{c} 0} \approx 0.5$.

Full evaluation of the on-chip HTS mixer has been carried out at temperatures from 20 to $77 \mathrm{~K}$. For clarity, only the results measured at a temperature of $40 \mathrm{~K}$ are presented in detail. Fig. 4 shows the conversion gain versus bias current $I_{\mathrm{B}}$ for a fixed RF signal $\left(f_{\mathrm{RF}}=7.2 \mathrm{GHz}\right.$ and $P_{\mathrm{RF}}=-55$ $\mathrm{dBm})$ and $\mathrm{LO}$ power. The operating range is quite broad but the maximum conversion gain $(\sim-8 \mathrm{~dB})$ is obtained at $I_{\mathrm{B}} \approx 50 \mu \mathrm{A}$. Two maxima of $P_{\mathrm{IF}}$ against $I_{\mathrm{B}}$ have been repeatedly observed of which the cause is not yet fully understood. Fig. 5 shows the conversion gain against the LO input power level with a maximum gain of $\sim-8 \mathrm{~dB}$ at $P_{\mathrm{LO}} \sim-32 \mathrm{dBm}$. The LO power for optimal operation is significantly lower than that required for pumping a semiconductor mixer, which is a major advantage of the Josephson mixer.

The frequency response of the on-chip HTS downconverter is shown in Fig. 6. The pass-band is between 6.7-8.5 GHz, which corresponds to that of the RF BPF's transmission response (a separate filter characterisation showed a

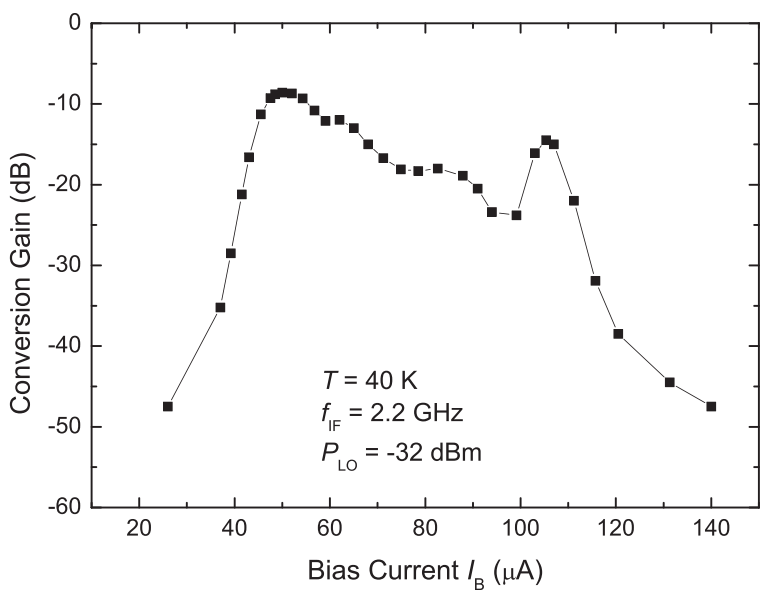

FIG. 4. Conversion gain versus bias current $I_{\mathrm{B}}$.

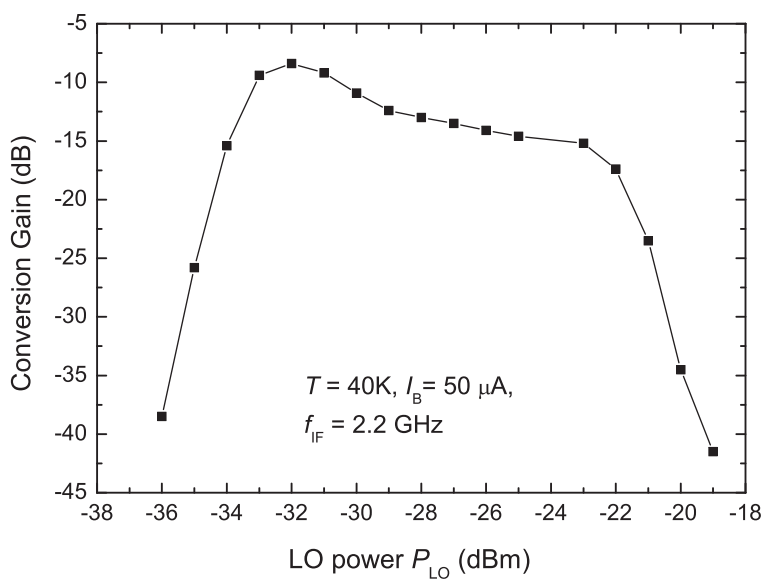

FIG. 5. IF output versus the LO input power.

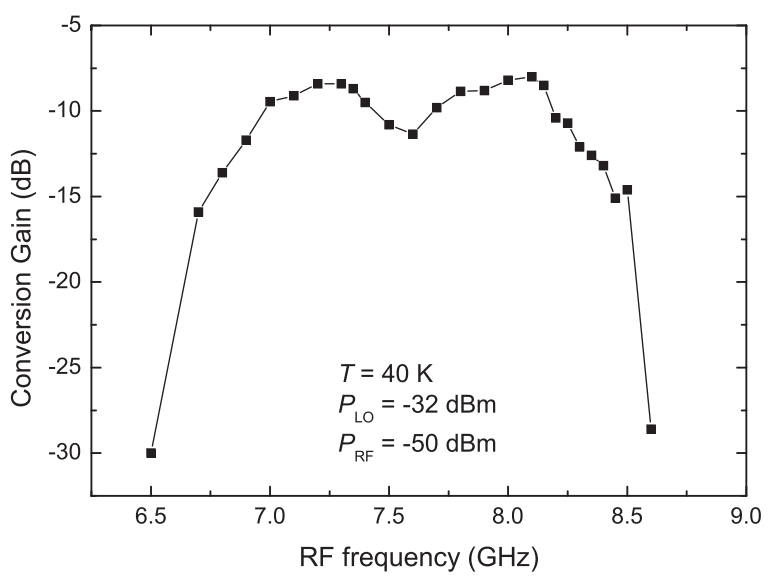

FIG. 6. Frequency response of the down-converter.

band-pass of $6.7-8.7 \mathrm{GHz}$ ). The maximum gain in band is around $-8 \mathrm{~dB}$. The drop at $\sim 7.6 \mathrm{GHz}$ is due to the unwanted interference between the expected spectrum $f_{R F}-f_{L O}$ and an unexpected spurious spectrum $2 f_{L O}-f_{R F}$. This can be eliminated by increasing LO frequency toward RF frequency in future design.

Fig. 7 shows the experimental result of the operating range and linearity. The IF output power increases linearly with the RF input power from $-80 \mathrm{dBm}$ to $-46 \mathrm{dBm}(1-\mathrm{dB}$ gain compression point) and peaks at $P_{\mathrm{RF}}=-38 \mathrm{dBm}$. 


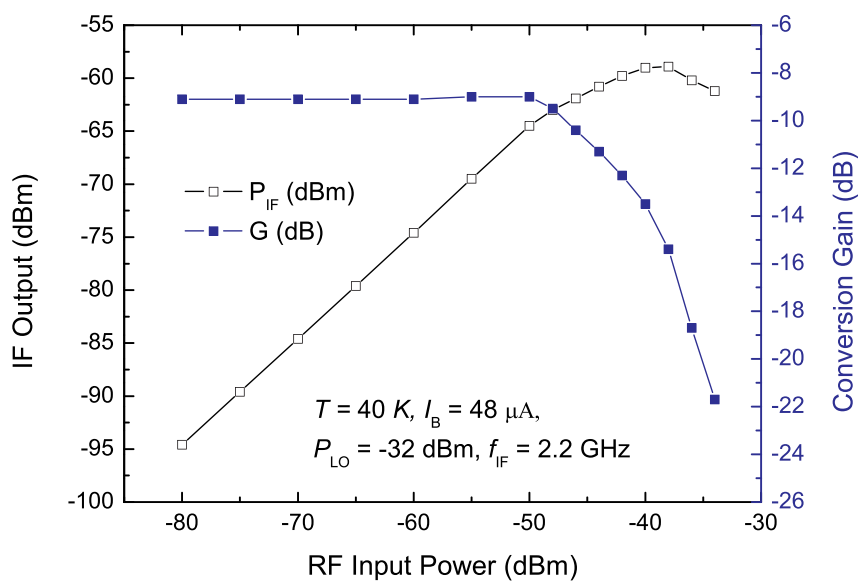

FIG. 7. IF output power and conversion gain as function of the input RF signal power at $\mathrm{T}=40 \mathrm{~K}$.

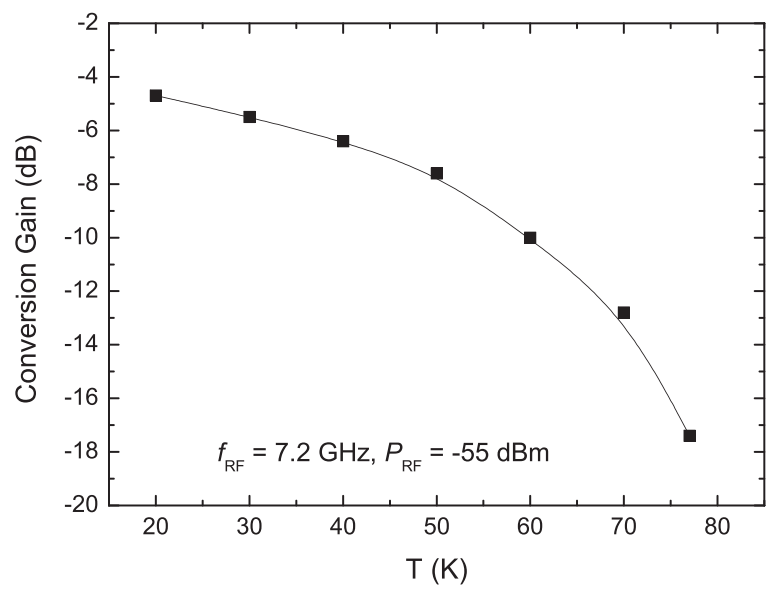

FIG. 8. Conversion gain versus operating temperature.

Further increase in the RF power causes the IF output to fall due to the junction overdriven by the RF power. Highly linear relationship between the IF output and the RF input was demonstrated, where the conversion gain maintains constant, an ideal mixer behaviour.

The HTS MMIC down-converter operated stably from $20 \mathrm{~K}$ to $77 \mathrm{~K}$. The best conversion gain obtained at each temperature is plotted in Fig. 8. For a fixed RF signal, $f_{\mathrm{RF}} \sim 7.2 \mathrm{GHz}$ and $P_{\mathrm{RF}}=-55 \mathrm{dBm}$, the bias $I_{\mathrm{B}}$ and LO power $P_{\text {LO }}$ were adjusted to yield the maximum conversion gain at each temperature. The conversion gain decreases with increasing the temperature; from $-4.7 \mathrm{~dB}$ (at $P_{\mathrm{LO}}=-31 \mathrm{dBm}$ ) at $20 \mathrm{~K}$ to $-12.8 \mathrm{~dB}$ (at $P_{\mathrm{LO}}=-35 \mathrm{dBm}$ ) at $70 \mathrm{~K}$, and $-17.4 \mathrm{~dB}$ (at $P_{\mathrm{LO}}=-36 \mathrm{dBm}$ ) at $77 \mathrm{~K}$. The temperature dependence can be explained by Eq. (1); the dynamic resistance, $R_{D}$, increases with the decreasing temperature, resulting in the increase in conversion efficiency. This is the highest conversion gain reported to date for an HTS Josephson down-converter at comparable frequencies and temperatures.

Significant improvement in the conversion gain and the linearity has been obtained in the current monolithic down-converter compared to that of the previous version based on a self-pumped R-SQUID heterodyne mixer ${ }^{6}$ (where the maximum conversion gain is $-15 \mathrm{~dB}$ at $30 \mathrm{~K}$ ). The results compare favourably to the early work by Suzuki et al. ${ }^{4}$ where a maximum conversion gain of $-14.5 \mathrm{~dB}$ was reported at
$15 \mathrm{~K}$ with an LO power of $-25 \mathrm{dBm}$. Besides, the present MMIC HTS chip is half the size $(20 \mathrm{~mm} \times 20 \mathrm{~mm})$ of the down-converter reported in Ref. 4. The maximum conversion gain (or minimum conversion loss) is better than or comparable to the best semiconductor mixers without amplification ${ }^{19}$ but the HTS mixer uses significantly lower LO power. The high conversion efficiency obtained here can be attributed to the following reasons: (1) high junction $R_{\mathrm{n}}$ value improves the coupling of RF power into the junction, (2) high dynamic resistance $R_{\mathrm{D}}$ value increases the IF output, (3) on-chip HTS filters and resonator provides effective RF signal coupling, isolation, and prevention of leakage between RF, LO, and IF ports, and (4) low-loss connections between the components.

In summary, a compact HTS MMIC down-converter, consisting of an externally pumped YBCO step-edge Josephson mixer integrated with several HTS passive components, is demonstrated. The integration of the HTS filters and resonator with the Josephson mixer improves the coupling efficiency, provides good isolation between RF, IF, and LO signals and reduces the loss along the transmission lines. The high $R_{\mathrm{n}}$ and $R_{\mathrm{D}}$ values of the step-edge junction improve the RF input coupling efficiency and the IF output efficiency. Stable operation was obtained between $20 \mathrm{~K}$ and $77 \mathrm{~K}$ with a maximum conversion gain of $-4.7 \mathrm{~dB}$ at $20 \mathrm{~K}$, the highest conversion gain reported for HTS mixers. The results demonstrated the potential of the HTS technology for application in wireless communication systems.

We acknowledge technical assistance from Jeina Lazar, Peter Sullivan, and Mei Shen of CSIRO; and earlier contribution and advice from Dr. John Macfarlane of CSIRO and Dr. Colin Pegrum of University of Strathclyde.

${ }^{1}$ R. R. Mansour, IEEE Trans. Microwave Theory Tech. 50, 750 (2002).

${ }^{2}$ R. W. Simon, R. B. Hammond, S. J. Berkowitz, and B. A. Willemsen, Proc. IEEE 92, 1585 (2004).

${ }^{3}$ N. Klein, Rep. Prog. Phys. 65, 1387 (2002).

${ }^{4}$ K. Suzuki, S. Yoshikawa, K. Yamaguchi, K. Hayashi, S. Fujino, T. Takenaka, T. Mitsuzuka, Y. Enomoto, K. Imai, F. Suginoshita, and N. Yazawa, IEEE MTT-S 1993 Int. Microwave Symposium Digest (IEEE, 1993), Vol. 3, p. 1429.

${ }^{5}$ L. Hao, J. Gallop, and J. Macfarlane, J. Supercond. Novel Magn. 19, 591 (2007).

${ }^{6}$ J. Du, T. Zhang, J. C. Macfarlane, Y. J. Guo, and X. W. Sun, Appl. Phys. Lett. 100, 262604 (2012).

${ }^{7}$ J. C. Macfarlane, J. Du, R. Taylor, and C. M. Pegrum, IEEE Trans. Appl. Supercond. 19, 920 (2009).

${ }^{8}$ J. Du and J. C. Macfarlane, Electron. Lett. 47, 772 (2011).

${ }^{9}$ J. Du, J. C. Macfarlane, T. Zhang, Y. Cai, and Y. J. Guo, Supercond. Sci. Technol. 25, 025019 (2012).

${ }^{10}$ J. Du, J. C. Macfarlane, C. M. Pegrum, T. Zhang, Y. Cai, and Y. J. Guo, J. Appl. Phys. 111, 053910 (2012).

${ }^{11}$ T. Zhang, J. Du, Y. J. Guo, and X. W. Sun, Electron. Lett. 48(12), 729 (2012).

${ }^{12}$ T. Zhang, J. Du, Y. J. Guo, and X. W. Sun, Supercond. Sci. Technol. 25, 105014 (2012).

${ }^{13}$ T. Van Duzer and C. W. Turner, Superconductive Devices and Circuits 2nd ed. (Prentice-Hall, Inc., 1999), Chap. 5.

${ }^{14}$ K. Yamaguchi, A. Kawaji, K. Suzuki, Y. Enomoto, and S. Tanaka, Electron. Commun. Jpn., Part 2 80, 69 (1997).

${ }^{15}$ Y. Taur, J. H. Claassen, and P. L. Richards, Appl. Phys. Lett. 24, 101 (1974).

${ }^{16}$ D. P. Butler, W. Yang, J. Wang, A. Bhandari, and Z. Celik-Butler, Appl. Phys. Lett. 61, 333 (1992).

${ }^{17}$ C. P. Foley, E. E. Mitchell, S. K. H. Lam, B. Sankrithyan, Y. M. Wilson, D. L. Tilbrook, and S. J. Morris, IEEE Trans. Appl. Supercond. 9, 4281 (1999).

${ }^{18}$ E. E. Mitchell and C. P. Foley, Supercond. Sci. Technol. 23, 065007 (2010).

${ }^{19}$ H. K. Chiou and T. Y. Yang, IEEE Trans. Microwave Theory Tech. 56, 835 (2008). 